\title{
Negative high-frequency differential conductivity in semiconductor superlattices
}

\author{
Yuriy A. Romanov \\ Institute for Physics of Microstructures RAS, 603600 Nizhny Novgorod, \\ Russia \\ Lev G. Mourokh and Norman J.M. Horing \\ Department of Physics and Engineering Physics, \\ Stevens Institute of Technology, Hoboken, NJ 07030
}

(October 28, 2018)

\begin{abstract}
We examine the high-frequency differential conductivity response properties of semiconductor superlattices having various miniband dispersion laws. Our analysis shows that the anharmonicity of Bloch oscillations (beyond tightbinding approximation) leads to the occurrence of negative high-frequency differential conductivity at frequency multiples of the Bloch frequency. This effect can arise even in regions of positive static differential conductivity. The influence of strong electron scattering by optic phonons is analyzed. We propose an optimal superlattice miniband dispersion law to achieve highfrequency field amplification.
\end{abstract}




\section{Introduction}

Semiconductor superlattices have been at the focal point of research in the last few decades because of their unique electronic properties. Their additional spatial periodicity leads to the formation of narrow Brillouin minizones $\left(10^{5}-10^{7} \mathrm{~cm}^{-1}\right)$ and energy minibands $\left(10^{-3}-10^{-1} \mathrm{eV}\right)[1,2,3]$. Due to the narrowness of these minibands Bloch oscillations [4] can be observed to occur even in relatively weak static electric fields $\left(10^{2}-10^{4} \mathrm{~V} / \mathrm{cm}\right)$ underscoring the promise of semiconductor superlattices as a likely mechanism for the amplification of $\mathrm{THz}$ signals. The occurence of Bloch oscillations was confirmed experimentally in a number of works [5].

It is well-known [6, [7,8] that within the tight-binding approximation a superlattice subject to a static electric field with strength $E_{c}$ can only amplify fields having frequencies $\omega<\sqrt{\Omega_{c}^{2}-\tau^{-2}}$, where $\Omega_{c}=e E_{c} d / \hbar, d$ is the superlattice period, and $\tau$ is the relaxation time. Moreover, amplification is only possible in the region of current-voltage characteristics having negative static differential conductivity $\left(\Omega_{c} \tau>1\right)$. For $\omega \tau>>1$ the real part of high-frequency conductivity, $\sigma(\omega)$, has a characteristic resonant structure, with its maxi-

mal negative value at frequency $\omega=\Omega_{c} \sqrt{1-\left(\Omega_{c} \tau\right)^{-2}-2\left(\Omega_{c} \tau\right)^{-1}}$, i.e. near $\Omega_{c}$. The origin of such behavior of the high-frequency conductivity, associated with the resonant interaction of Bloch oscillations with the external time-dependent field, as well as with electron bunching in momentum space, was discussed in Ref. [7] in detail. In the Wannier-Stark representation [9], this resonant character is an obvious consequence of energy conservation in electron transport (transfer along the Wannier-Stark ladder) accompanied by photon emission/absorption [10].

Further consideration beyond the tight-binding approximation, leading to anharmonicity of Bloch oscillations, can exhibit drastic change in the character of amplification of timedependent fields. In particular, the resonant interaction of Bloch oscillation harmonics with the external field can give rise to the amplification of harmonic fields with frequencies $\omega \sim \nu \Omega_{c},(\nu$ is an integer $)$. Such amplification can occur even in regions of current-voltage characteristics having positive static differential conductivity. This is extremely important in connection with the necessity of low-frequency domain instability suppression [5].

The object of the present paper is the analysis of electron high-frequency behavior in superlattices having various miniband dispersion laws, and the exploration of new phenomenology brought about by the anharmonicity of Bloch oscillations. We compare the following two miniband dispersion laws:

1. The commonly used "sinusoidal" dispersion law in the tight-binding approximation:

$$
\varepsilon_{3}\left(k_{3}\right)=\frac{\Delta}{2}\left(1-\cos \left(k_{3} d\right)\right)
$$

where $\varepsilon_{3}$ and $k_{3}$ are electron energy and wave vector along the superlattice axis, respectively, and $\Delta$ is the miniband width. In this case there is the region with negative effective electron mass in the upper half of miniband.

2.The model "parabolic" dispersion law with no regions of negative effective mass, but with Bragg reflections at points $k_{3}= \pm \pi / d$ : 


$$
\varepsilon_{3}\left(k_{3}\right)=\frac{\hbar^{2} k_{3}^{2}}{2 m_{3}}, \quad-\frac{\pi}{d}<k_{3}<\frac{\pi}{d} .
$$

This article is structured as follows. In Section II we derive an expression for highfrequency differential conductivity which is valid for any miniband dispersion law. The high-frequency electron dynamics for miniband dispersion laws given by Eq.(1) and Eq.(2) are compared in Section III without scattering by optic phonons; and in Section IV they are compared in the case of strong electron-phonon interaction. In Section V we suggest a possible miniband dispersion law having properties desirable for time-dependent field amplification and analyze the high-frequency electron dynamics for this case. Section VI presents the conclusions of our work.

\section{General Relations}

To analyze the high-frequency differential conductivity, we will determine the induced superlattice current for an arbitrary miniband dispersion law in the presence of an external electric field given by

$$
E(t)=E_{c}+E_{1} \cos \omega t
$$

To start, we employ a Boltzmann equation with a single relaxation time approximation:

$$
\frac{\partial f(\mathbf{k}, t)}{\partial t}+\frac{e \mathbf{E}(t)}{\hbar} \frac{\partial f(\mathbf{k}, t)}{\partial \mathbf{k}}=\frac{f(\mathbf{k}, t)-f_{0}(\varepsilon, T)}{\tau}
$$

where $f(\mathbf{k}, t)$ and $f_{0}(\varepsilon, T)$ are the nonequilibrium and equilibrium distribution functions, respectively, and $T$ is the lattice temperature. The electric field $\mathbf{E}$ is applied along the superlattice axis. Using periodicity in $\mathbf{k}$-space, we expand functions of interest in Fourier series:

$$
\begin{gathered}
\varepsilon(\mathbf{k})=\sum_{\nu=-\infty}^{\infty} \varepsilon\left(\nu, k_{\perp}\right) \exp \left(i \nu k_{3} d\right), \\
f_{0}(\varepsilon, T)=\sum_{\nu=-\infty}^{\infty} F_{\nu}\left(k_{\perp}\right) \exp \left(i \nu k_{3} d\right),
\end{gathered}
$$

and

$$
f(\mathbf{k}, t)=\sum_{\nu=-\infty}^{\infty} F_{\nu}\left(k_{\perp}\right) \exp \left(i \nu k_{3} d\right) \Phi_{\nu}(t)
$$

where $F_{\nu}$ is the Fourier coefficient of the equilibrium distribution function,

$$
F_{\nu}\left(k_{\perp}\right)=\frac{d}{2 \pi} \int_{-\pi / d}^{\pi / d} f_{0}(k) \exp \left(-i \nu k_{3} d\right), \quad F_{\nu}=F_{-\nu}^{*}
$$


and $\Phi_{\nu}$ is the factor by which the Fourier transform of the nonequilibrium distribution function differs from its equilibrium counterpart, $F_{\nu}\left(k_{\perp}\right.$ is the component of electron wave vector orthogonal to the superlattice axis). Substituting Eq.(7) into Eq.(4), we obtain the kinetic equation for the multicomponent function $\Phi_{\nu}(t)$ as

$$
\tau \frac{d \Phi_{\nu}(t)}{d t}+(1+i \nu \tau \Omega(t)) \Phi_{\nu}(t)=1, \quad \Omega(t)=\frac{e d E(t)}{\hbar}
$$

with the initial condition

$$
\Phi_{\nu}(0)=1
$$

The average superlattice current can be expressed in terms of the functions $\Phi_{\nu}(t)$ as

$$
j(t)=\frac{i}{2} \sum_{\nu=1}^{\infty} j_{0 \nu} \Phi_{\nu}(t)+c . c .
$$

where

$$
j_{0 \nu}=-\frac{4 e d}{\hbar} \nu \int \frac{d^{3} k}{(2 \pi)^{3}} F_{\nu}\left(k_{\perp}\right) \varepsilon(k) \exp \left(i \nu k_{3} d\right), \quad j_{0 \nu}^{*}=-j_{0,-\nu} .
$$

Solving Eq.(9) with the electric field of Eq.(3) $\left(e E_{1} d / \hbar \omega<<1\right)$, we obtain the complex linear differential superlattice conductivity at frequency $\omega$ in the presence of a static field $E_{c}$ as

$$
\begin{aligned}
\sigma\left(\omega, \Omega_{c}\right)= & \sum_{\nu=1}^{\infty} \frac{\sigma_{0 \nu}}{\left(1+\left(\nu \Omega_{c} \tau\right)^{2}-(\omega \tau)^{2}\right)^{2}+4(\omega \tau)^{2}} \\
& \times\left(1-\left(\nu \Omega_{c} \tau\right)^{2}+(\omega \tau)^{2}+i \omega \tau \frac{1-3\left(\nu \Omega_{c} \tau\right)^{2}+(\omega \tau)^{2}}{1+\left(\nu \Omega_{c} \tau\right)^{2}}\right)
\end{aligned}
$$

where

$$
\sigma_{0 \nu}=\frac{e d \tau}{\hbar} \nu j_{0 \nu}
$$

It should be noted that this expression is valid for any miniband dispersion law (which is involved in Eq.(12) and, consequently, in Eq.(13)).

\section{High-frequency electron conductivity}

In this Section we apply the general miniband results obtained above to the two miniband dispersion laws given by Eq.(1) and Eq.(2). Substituting Eq.(1) and Eq.(2) into Eq.(12), we obtain

$$
j_{0 \nu}=\delta_{\nu 1} j_{0}, \quad j_{0}=\frac{e n d}{\hbar}\left(\frac{\Delta}{2}-\left\langle\varepsilon_{3}\right\rangle_{0}\right)=\frac{e n d \Delta}{2 \hbar} I_{\nu}\left(\frac{\Delta}{2 T}\right) I_{0}^{-1}\left(\frac{\Delta}{2 T}\right)
$$


for the "sinusoidal" dispersion law and

$$
j_{0 \nu}=(-1)^{\nu+1} \frac{2 \hbar n e}{m d \nu} \exp \left(-\frac{\pi^{2} T}{4 \Delta} \nu^{2}\right), \quad \Delta=\frac{\hbar^{2} \pi^{2}}{2 m d^{2}}
$$

for the "parabolic" one. Here, $n$ is the electron concentration, $\left\langle\varepsilon_{3}\right\rangle_{0}$ is the average equilibrium longitudinal electron energy, and $I_{\nu}(x)$ is the modified Bessel function. The second equality in Eq.(15) is given for nondegenerate Maxwellian statistics with arbitrary $T$, and Eq.(16) is given for Maxwellian statistics with $T<\Delta$.

Accordingly, for superlattices having a "sinusoidal" dispersion law, Bloch oscillations involve the fundamental harmonic alone, $\sigma_{0 \nu}=\sigma_{0} \delta_{1 \nu}$ ( $\sigma_{0}$ is the static superlattice conductivity), and Eq.(13) is in agreement with results of Refs. [6.8]. However, for a "nonsinusoidal" miniband dispersion law (reflecting the possibility of electron transport not only to nearby cells) the terms with $\nu>1$ also contribute to the conductivity (13). Their contribution can be obtained by replacing of $\Omega_{c}$ by $\nu \Omega_{c}$. Formally, this replacement is equivalent to an increase of the superlattice period by the factor $\nu$, and, consequently, to a decrease of the Brillouin miniband wavenumber range by a factor $1 / \nu$. To illustrate this behavior, we use the "parabolic" dispersion law, given by Eq.(2). In this case, according to Eq.(16), we have for $T \rightarrow 0$

$$
\sigma_{0 \nu}=(-1)^{\nu+1} 2 \sigma_{0}
$$

Substituting Eq.(17) into Eq.(13) and performing the summation with respect to $\nu$, we obtain the real part of the high-frequency differential conductivity as

$$
\operatorname{Re} \sigma\left(\omega, \Omega_{c}\right)=\sigma_{0}\left(\frac{1}{1+(\omega \tau)^{2}}-\frac{\pi \cosh \left(\pi / \Omega_{c} \tau\right) \sin \left(\pi \omega / \Omega_{c}\right)}{\Omega_{c} \omega \tau^{2}\left(\sinh ^{2}\left(\pi / \Omega_{c} \tau\right)+\sin ^{2}\left(\pi \omega / \Omega_{c}\right)\right)}\right)
$$

For $\omega>\Omega_{c}>>\tau^{-1}$, Eq.(18) can be rewritten as

$$
\operatorname{Re} \sigma\left(\omega, \Omega_{c}\right)=-\sigma_{0} \frac{\left(\Omega_{c} / \pi \omega\right) \sin \left(\pi \omega / \Omega_{c}\right)}{1+\left(\Omega_{c} \tau / \pi\right)^{2} \sin ^{2}\left(\pi \omega / \Omega_{c}\right)} .
$$

It follows from Eq.(19) that the real part of the high-frequency differential conductivity changes its sign at $\omega \sim \nu \Omega_{c}$. For $\omega \tau<<1$, Eq.(18) limits to the static differential conductivity relation

$$
\sigma\left(0, \Omega_{c}\right)=\sigma\left(1-\left(\pi / \Omega_{c} \tau\right)^{2} \frac{\cosh \left(\pi / \Omega_{c} \tau\right)}{\sinh ^{2}\left(\pi / \Omega_{c} \tau\right)}\right)
$$

which is negative for $\Omega_{c} \tau>1.174$. The conductivity of Eq.(18) is depicted in Figure 1(a) for $\Omega_{c} \tau=1.15,2$, and 5 , respectively. For comparison, we present the conductivity involving the "sinusoidal" dispersion law in the Insert. Figure 1(b) clearly shows the resonant character of the high-frequency superlattice differential conductivity for the (unrealistic) high value $\Omega_{c} \tau=30$. It is evident from the Figures and from Eq.(19) that the high-frequency superlattice differential conductivity is negative near the Bloch oscillation harmonics, at frequencies $\omega<(2 \nu+1) \Omega_{c}$ and $\omega>2 \nu \Omega_{c}$. This behavior is a consequence of the sign of the Fourier 
coefficients of the "parabolic" dispersion law, as reflected in Eq.(17). One can see that, for superlattices having a "nonsinusoidal" miniband dispersion law, amplification of external time-dependent fields is possible for frequencies larger than the Bloch frequency, near its harmonics and even (most importantly) in the regions of the current-voltage characteristic having positive static differential conductivity.

\section{Phonon effects on high-frequency electron dynamics}

In semiconductor structures having strong electron coupling to optic phonons Bragg reflection may be pre-empted by the emission of a phonon with the return of the electron to the bottom of the miniband. For simplicity, we assume that the miniband width, $\Delta$, is equal to or a little larger than the phonon energy, $\hbar \omega_{0}$, and that the characteristic time of phonon emission, $\tau_{0}$, is shorter than all other relaxation times. If scattering is absent in the passive region (the region of energies with $\varepsilon<\hbar \omega_{0}$ ), the electron motion in momentum space is periodic with frequency $2 \Omega_{c}$, and in coordinate space there are oscillations with the same frequency as well as a drift with velocity

$$
\langle V\rangle=\frac{d}{\pi \hbar} \int_{0}^{\pi / d} \frac{\partial \varepsilon\left(k_{3}\right)}{\partial k_{3}} d k_{3} .
$$

In this case the electron momentum distribution function becomes needle-like (sharply peaked in the $k_{3}$-direction, "streaming") and the problem is really one-dimensional. It should be noted that, in contrast to the case of a bulk semiconductor, for a superlattice the extent of electron penetration in the active region (the region of energies with $\varepsilon>\hbar \omega_{0}$ ) is determined not only by the phonon emission time, but by the miniband width as well. As a result, streaming can be much narrower in superlattices than in bulk semiconductors. Furthermore, if $\tau_{0}$ is not very small, there is a finite probability, $(1-\alpha)$, of Bragg reflection before phonon emission. In this case the electron oscillations have two characteristic transfer frequencies, $\Omega_{c}$ and $2 \Omega_{c}$, and the electron distribution function is again needle-like, but is peaked not only for positive $k_{3}$ but also for negative $k_{3}$. The new cyclic electron motion with frequency $2 \Omega_{c}$ can result in high-frequency negative differential conductivity at frequencies close to $2 \nu \Omega_{c}$ ( $\nu$ is an integer). The occurrence of such a negative conductivity in bulk semiconductors was predicted and studied theoretically in Ref. [11] and was confirmed experimentally in Ref. [12]. It is a consequence of (a) electron bunching in momentum space near the involved part of the $k_{3}$-axis under the influence of a strong static electric field with optical phonon scattering, and (b) modulation of the electron momentum distribution by the additional harmonic field jointly with relaxation processes. The necessary conditions for such negative conductivity are relatively weak electron scattering in the passive region $\left(\Omega_{c} \tau>1\right)$ and small depth of penetration in the active region. In summary, streaming in superlattices has the following features that distinguish it from bulk: 1) the electron penetration depth in the active region is determined by the miniband width independently of the phonon emission time; 2) streaming occurs for both positive and negative $k_{3}$ (double-side 
streaming) and there are two characteristic transfer frequencies in momentum space; 3) the dependence of electron velocity on its momentum is nonlinear.

Electron dynamics in the passive region are described by the Boltzmann equation (Eq.(4)) with the boundary condition

$$
f\left(-\frac{\pi}{d}\right)=(1-\alpha) f\left(\frac{\pi}{d}\right)
$$

In the interest of simplicity, we take the initial electron distribution function in the form

$$
f_{0}\left(k_{3}\right)=2 \pi n \delta\left(k_{3}\right)
$$

For convenience, we employ notation with an additional argument inserted into all functions, the inverse relaxation time, $\tau^{-1}$. Assuming the field strength $E_{1}$ in Eq.(3) to be much smaller than the static field strength $E_{c}$, we write

$$
f\left(k_{3}, t, \tau^{-1}\right)=f_{c}\left(k_{3}, \tau^{-1}\right)+f_{1}\left(k_{3}, t, \tau^{-1}\right),
$$

where the static nonequilibrium distribution function $f_{c}\left(k_{3}, \tau^{-1}\right)$ is given by

$$
\begin{aligned}
& f_{c}\left(k_{3}, \tau^{-1}\right)=\frac{2 \pi n d}{\Omega_{c} \tau} \frac{\exp \left(-\frac{k_{3} d}{\Omega_{c} \tau}\right)}{(1-}\left.\exp \left(-\frac{\pi}{\Omega_{c} \tau}\right)\right)\left(1+(1-\alpha) \exp \left(-\frac{\pi}{\Omega_{c} \tau}\right)\right) \\
& \times\left\{\begin{array}{c}
1, \quad 0<k_{3}<\pi / d \\
(1-\alpha) \exp \left(\frac{2 \pi}{\Omega_{c} \tau}\right),-\pi / d<k_{3}<0,
\end{array}\right.
\end{aligned}
$$

and $f_{1}\left(k_{3}, t, \tau^{-1}\right)=f_{1}\left(k_{3}, \tau^{-1}\right) \exp (-i \omega t)$ obeys the linearized Boltzmann equation

$$
e E_{c} \frac{\partial f_{1}\left(k_{3}, \tau^{-1}\right)}{\hbar \partial k_{3}}+e E_{1} \frac{\partial f_{c}\left(k_{3}, \tau^{-1}\right)}{\hbar \partial k_{3}}=-\left(\tau^{-1}-i \omega\right) f_{1}\left(k_{3}, \tau^{-1}\right),
$$

with the boundary condition of Eq.(21) and the particle conservation condition

$$
\int_{-\pi / d}^{\pi / d} f_{1}\left(k_{3}, \tau^{-1}\right) \frac{d k_{3}}{2 \pi}=0
$$

The static distribution function of Eq.(24) facilitates the determination of static currentvoltage characteristics. For the "sinusoidal" miniband dispersion law, it is given by

$$
j_{c}=\tilde{j}_{0} \frac{\Omega_{c} \tau}{1+\left(\Omega_{c} \tau\right)^{2}} \frac{1-(1-\alpha) \exp \left(-\pi / \Omega_{c} \tau\right)}{1+(1-\alpha) \exp \left(-\pi / \Omega_{c} \tau\right)} \operatorname{coth}\left(\frac{\pi}{2 \Omega_{c} \tau}\right)
$$

whereas in the case of the "parabolic" miniband dispersion law, we have

$$
j_{c}=\tilde{j}_{0}\left(\frac{2}{\pi}\right)^{2}\left(\Omega_{c} \tau-\frac{(2-\alpha) \pi}{2 \sinh \left(\pi / \Omega_{c} \tau\right)-\alpha\left(1-\exp \left(-\pi / \Omega_{c} \tau\right)\right)}\right) .
$$

Here, $\tilde{j}_{0}=n e \Delta d / \hbar$. It is important to note that in the case of high probability of phonon emission, $\alpha \sim 1$, the negative static differential conductivity is suppressed and can even 
vanish for any miniband dispersion law [13]. However, in the present paper we are primarily interested in high-frequency electronic properties of superlattices and, accordingly, in the distribution function $f_{1}\left(k_{3}, t, \tau^{-1}\right)$. The solution of Eq.(25) for this function can be represented in the form

$$
f_{1}\left(k_{3}, t, \tau^{-1}\right)=\frac{i}{\omega \tau} \frac{E_{1}}{E_{c}}\left(f_{c}\left(k_{3}, \tau^{-1}\right)-f_{c}\left(k_{3}, \tau^{-1}-i \omega\right)\right) .
$$

Consequently, the high-frequency conductivity is related to static conductivity (in the single $\tau$-approximation considered here), as given by

$$
\sigma\left(\omega, \tau^{-1}\right)=\frac{i}{\omega \tau}\left(\sigma_{c}\left(\tau^{-1}\right)-\sigma_{c}\left(\tau^{-1}-i \omega\right)\right)
$$

It should be emphasized that the closeness of static conductivity values for different miniband dispersion laws does not imply closeness of the corresponding high-frequency conductivities. This is clear from Eq.(30), since the replacement of relatively small real frequencies $\left(\tau^{-1}\right)$ by relatively large complex values $\left(\tau^{-1}-i \omega\right)$ leads to the occurrence of resonant terms and significant phase shifts between current and applied field.

Only the second terms on the right sides of Eqs. $(29,30)$ contribute to the real part of the high-frequency conductivity. It is evident from Eqs. $(24,29)$ that the corresponding part of the distribution function $f_{1}\left(k_{3}, t, \tau^{-1}\right)$ contains a modulation factor $\exp \left\{i\left(\omega d k_{3} / \Omega_{c}-\omega t\right)\right\}$ describing an electron density wave moving in momentum space with velocity $e E_{c}$ and wavelength $\lambda=2 \pi \Omega_{c} / \omega d$. There is a resonant energy exchange between the electron density wave and the applied harmonic field occuring at frequencies close to the transfer frequency harmonics, $\omega \sim 2 \nu \Omega_{c}$ for single-side streaming, and $\omega \sim(2 \nu+1) \Omega_{c}$ for double-side streaming. In these cases there is an approximately integer number of wave lengths $\lambda$ in corresponding transfer regions, $\pi / d$ or $2 \pi / d$, respectively.

The expressions for the high-frequency conductivities are extremely unwieldy in the general case and we present them only for $\alpha=1$. For the "sinusoidal" miniband dispersion law, the high-frequency conductivity has the form

$$
\operatorname{Re} \sigma(\omega)=\sigma_{0} \frac{\left(1+(\omega \tau)^{2}-\left(\Omega_{c} \tau\right)^{2}\right) \sinh \left(\pi / \Omega_{c} \tau\right)+\left(1+(\omega \tau)^{2}+\left(\Omega_{c} \tau\right)^{2}\right)(\omega \tau)^{-1} \sin \left(\pi \omega / \Omega_{c}\right)}{\left(\left(1-(\omega \tau)^{2}+\left(\Omega_{c} \tau\right)^{2}\right)^{2}+4(\omega \tau)^{2}\right)\left(\cosh \left(\pi / \Omega_{c} \tau\right)-\cos \left(\pi \omega / \Omega_{c}\right)\right)}
$$

whereas for the "parabolic" miniband dispersion law it is given by

$$
\sigma(\omega)=\sigma_{0}\left(\frac{1+i \omega \tau}{1+(\omega \tau)^{2}}-\frac{\pi}{2 \Omega_{c} \omega \tau^{2}} \frac{\sin \left(\pi \omega / \Omega_{c}\right)+2 i \operatorname{coth}\left(\pi / 2 \Omega_{c} \tau\right) \sin ^{2}\left(\pi \omega / \Omega_{c}\right)}{\cosh \left(\pi / 2 \Omega_{c} \tau\right)-\cos \left(\pi \omega / \Omega_{c}\right)}\right) .
$$

The real parts of the conductivities of Eqs.(31,32) are presented in Figure 2(a) and Figure 2(b), respectively. In the first case (Fig.2(a)), there is no negative high-frequency differential conductivity at any frequency. At resonant frequencies, $\omega=2 \nu \Omega_{c}$, the highfrequency conductivity has maxima which do not depend on $\tau$ for $\Omega_{c} \tau \rightarrow \infty$ (relaxation is only due to phonon emission at the miniband edge) and are approximately given by

$$
\sigma(\omega) \sim \frac{2 \tilde{j}_{0}}{\pi\left(4 \nu^{2}-1\right) E_{c}}
$$


In the second case, (Fig.2(b)), the high-frequency conductivity changes sign as $\omega$ passes through the resonant frequencies, and in their vicinities (at $\omega \sim 2 \nu \Omega_{c} \pm \tau^{-1}$ ) it has the form

$$
\sigma(\omega) \sim \mp \frac{2}{5} \frac{\tilde{j}_{0}}{5 \pi^{2} \nu E_{c}},
$$

which, also, does not depend on $\tau$. The real and imaginary parts of the high-frequency conductivity for a superlattice calculated from Eqs. $(28,30)$, are presented in Figures $3(\mathrm{a})$ and 3(b), respectively, for $\Omega_{c} \tau=10$ and various values of $\alpha$. Near the even harmonics $\left(2 \nu \Omega_{c}\right)$ both real and imaginary parts of the high-frequency conductivity are almost independent of $\alpha$, because (for "parabolic" case only!) the amplitudes of even Fourier-harmonics of the electron velocity do not depend on $\alpha$. In the vicinity of odd harmonics $\left((2 \nu+1) \Omega_{c}\right)$ there is resonant response only for small $\alpha$. It should be noted that the imaginary part of the high-frequency conductivity has large negative values which can lead to plasmon instability in superlattices near the transfer frequencies.

It is of value to understand the reasons for the strong dependence of high-frequency superlattice conductivity on the miniband dispersion law. The time-dependent current arises from joint modulation of the electron distribution function in momentum space by the timedependent field, Bragg reflection and scattering. The modulation associated with the timedependent field is a result of a homogeneous shift of electrons along the involved part of the $k_{3}$-axis. For this modulation to be nonzero, it is necessary that the shifted electron distribution in momentum space (created by the strong static field and Bragg reflection) should be inhomogeneous. In particular, without scattering in the passive region and with $\alpha=1, f_{c}\left(k_{3}\right)$ is constant and there is no modulation. Furthermore, to induce large current oscillations, the electron velocity must strongly depend on momentum. And, finally, to generate negative differential conductivity, the current phase has to be shifted with respect to the field phase by more than $\pi / 2$. To examine all these conditions for superlattices, we consider the region of resonant frequencies, $\omega=2 \nu \Omega_{c}+\delta \omega,|\delta \omega|<<\Omega_{c}$, and strong static fields, i.e. $\Omega_{c} \tau>>1$. For simplicity we will analyze single-side streaming, so we assume $\alpha=1$. In this case, according to Eqs. $(24,29)$, the part of the electron distribution function responsible for the real part of the high-frequency differential conductivity is given by

$$
\delta f_{1}\left(k_{3}\right) \approx \frac{2 n E_{1} d}{E_{c}\left(1+(\tau \delta \omega)^{2}\right)}\left[\tau \delta \omega \sin \left(2 \nu k_{3} d\right)-\cos \left(2 \nu k_{3} d\right)\right] \equiv \delta f_{1}^{(a)}\left(k_{3}\right)+\delta f_{1}^{(s)}\left(k_{3}\right) .
$$

The first term in the square brackets of Eq. $(35), \delta f_{1}^{(a)}\left(k_{3}\right)$, is antisymmetric with respect to the center of the transfer region (the point $k_{3}=\pi / 2 d$ ), whereas the second term, $\delta f_{1}^{(s)}\left(k_{3}\right)$, is symmetric. We can also rewrite the electron velocity as a sum of symmetric and antisymmetric parts, $V\left(k_{3}\right)=V_{a}\left(k_{3}\right)+V_{s}\left(k_{3}\right)$. In this notation, the real part of the high-frequency differential conductivity has the form

$$
\begin{aligned}
\operatorname{Re} \sigma(\omega) & \approx \frac{n e d}{\pi E_{c}\left(1+(\tau \delta \omega)^{2}\right)}\left[\tau \delta \omega \int_{0}^{\pi / d} V_{a}\left(k_{3}\right) \sin \left(2 \nu k_{3} d\right) d k_{3}-\int_{0}^{\pi / d} V_{s}\left(k_{3}\right) \cos \left((2 \nu+1) k_{3} d\right)\right] \\
& \equiv \sigma_{a}(\omega)+\sigma_{s}(\omega) .
\end{aligned}
$$

The conductivity $\sigma_{a}(\omega)$, determined by the antisymmetric velocity component, changes sign as $\omega$ passes through the resonant frequencies, $2 \nu \Omega_{c}$, whereas the conductivity $\sigma_{s}(\omega)$, determined by the symmetric velocity component, does not change sign, and it is positive, 
as usual (when $V_{s}\left(k_{3}\right)$ increases with increasing $k_{3}$ near the bottom of the miniband and decreases near the top). For superlattices having the "sinusoidal" miniband dispersion law, $V\left(k_{3}\right)=V_{s}\left(k_{3}\right)$, and for the superlattices having the "parabolic" miniband dispersion law, $V\left(k_{3}\right)=V_{a}\left(k_{3}\right)+$ const. As a result, negative high-frequency differential conductivity occurs in the latter case and does not exist in the former case.

\section{Optimal miniband dispersion law for high-frequency field amplification}

In the preceding Section we showed that the value of the negative high-frequency differential conductivity at the transfer frequencies depends not only on the electron scattering mechanisms in the passive region and the depth of penetration into the active region, but it is also very sensitive to the miniband dispersion law. In particular, contrary to the usual expectation, the existence of a negative effective electron mass region actually militates against the occurrence of negative high-frequency conductivity. Minibands having much higher electron velocities in the top part of the band than those in the bottom part are optimal for negative high-frequency differential conductivity. In this case modulation of the electron distribution function in momentum space induces a large time-dependent current, and, moreover, the contribution of the symmetric velocity component to the high-frequency differential conductivity becomes negative at the resonant frequencies. To explore this, we consider the conductivity of a structure having a "superquadratic" miniband dispersion law, as given by

$$
\varepsilon\left(k_{3}\right)=\frac{\hbar^{2}}{2}\left\{\begin{array}{cl}
\frac{k_{3}^{2}}{m_{1}}, & 0<\left|k_{3}\right|<\frac{\pi}{2 d} \\
\frac{k_{3}^{2}}{m_{2}}-\frac{\pi}{d}\left(\frac{1}{m_{2}}-\frac{1}{m_{1}}\right) & \left(\left|k_{3}\right|-\frac{\pi}{4 d}\right), \frac{\pi}{2 d}<\left|k_{3}\right|<\frac{\pi}{d} .
\end{array}\right.
$$

In the region $0<\left|k_{3}\right|<\pi / 2 d$, electrons have a positive effective mass $m_{1}$ and in the region $\pi / 2 d<\left|k_{3}\right|<\pi / d$, they have a different positive effective mass $m_{2}$. Qualitatively

similar dispersion laws are found in hole quantum layers [14. For such a dispersion law, the symmetric and antisymmetric electron velocity components are given by (for $k_{3}>0$ )

$$
V_{a}\left(k_{3}\right)=\frac{\hbar}{2}\left(\frac{1}{m_{2}}+\frac{1}{m_{1}}\right)\left(k_{3}-\frac{\pi}{2 d}\right),
$$

and

$$
V_{s}\left(k_{3}\right)=\frac{\hbar}{2}\left(\frac{1}{m_{2}}-\frac{1}{m_{1}}\right)\left|k_{3}-\frac{\pi}{2 d}\right|+\frac{\hbar \pi}{2 m_{1} d} .
$$

Substituting Eqs.(38,39) into Eq.(36), we obtain

$$
\operatorname{Re} \sigma(\omega) \approx \frac{-\sigma_{0}}{4 \nu\left(1+(\tau \delta \omega)^{2}\right)}\left((1+\eta) \frac{\delta \omega}{\Omega_{c}}+\frac{1-(-1)^{\nu}}{\pi \nu} \frac{\eta-1}{\Omega_{c} \tau}\right),
$$


where $\eta=m_{1} / m_{2}$. It follows from Eq.(40) that, for $\eta>1$, the contribution of the symmetric velocity component to the high-frequency conductivity is negative, and, for $\eta<1$, it is positive. The case with $\eta=-1$ corresponds to the "sinusoidal" dispersion law. The real part of the high-frequency differential conductivity is presented in Figure 4 for various ratios of effective masses, and with $\Omega_{c} \tau=3$. It should be noted that negative high-frequency conductivity occurs even for $\eta<1$, but its magnitude is much smaller than that for $\eta>1$. For very large $\eta$, negative high-frequency conductivity arises even for small static fields $\left(\Omega_{c} \tau<1\right)$, when the low-frequency differential conductivity is large and positive. To illustrate this, we present the high-frequency differential conductivity of a superlattice having the miniband dispersion law of Eq.(37) for $\eta=10,30$, and 50 and $\Omega_{c} \tau=0.8$.

In the analysis above we did not take into consideration the field dependence of the probability of phonon emission, $\alpha$. Account of this dependence would lead to an additional modulation of the electron distribution in momentum space and some modification (but not vanishing!) of the range of negative high-frequency differential conductivity. It should be noted that interminiband tunneling could also give rise to the formation of negative high-frequency differential conductivity in the regions having positive static differential conductivity [15].

\section{Conclusions}

In summary, our analysis shows that the anharmonicity of Bloch oscillations in superlattices having a "nonsinusoidal" miniband dispersion law (beyond the tight-binding approximation) should lead to amplification of harmonic fields with frequencies that are multiples of the Bloch frequency. Such amplification can occur even in regions of the currentvoltage characteristics that have a positive static differential conductivity. We have compared high-frequency electron behavior in superlattices having (i) "sinusoidal" (Eq.(1)) and (ii) "parabolic" (Eq.(2)) miniband dispersion laws for the cases (a) without optic phonon scattering and (b) with strong electron-optic phonon coupling. We have obtained explicit expressions for the high-frequency differential conductivity in all these situations exhibiting the regions where it is negative. On this basis, we propose the "superquadratic" miniband dispersion law (Eq.(37)) as optimal for high-frequency field amplification. This dispersion law can be realized in a superlattice of quasi-two-dimensional hole layers.

\section{Acknowledgements}

The work of Yu.A.R. is supported by the Russian Foundation for Basis Research (Grant No. 01-02-16446) and by the Program "Low-Dimensional Quantum Structures" of Russian Academy of Science, L.G.M. and N.J.M.H. gratefully acknowledge support from the US Department of Defense, DAAD 19-01-1-0592. 


\section{FIGURES}

FIG. 1. High-frequency differential conductivity for the "parabolic" miniband dispersion law

(Eq.(2)); (a) for $\Omega_{c} \tau=1.15 ; 2 ; 5$, (b) for $\Omega_{c} \tau=30$. Inserts: high-frequency conductivity of the "sinusoidal" miniband dispersion law (Eq.(1)).

FIG. 2. High-frequency differential conductivity for single-side streaming with $\Omega_{c} \tau=3 ; 5 ; 10$; (a) for the "sinusoidal" miniband dispersion law; (b) for the "parabolic" miniband dispersion law.

FIG. 3. High-frequency differential conductivity for double-side streaming for the "parabolic" miniband dispersion law with $\Omega_{c} \tau=10$ and $\alpha=0 ; 0.5 ; 1$; (a) real part; (b)imaginary part.

FIG. 4. High-frequency differential conductivity for the "superquadratic" miniband dispersion law (Eq.(37)) with $\Omega_{c} \tau=3$ and $\eta=1 ; 5 ; 10$.

FIG. 5. High-frequency differential conductivity for the "superquadratic" miniband dispersion law with $\Omega_{c} \tau=0.8$ and $\eta=10 ; 30 ; 50$. 


\section{REFERENCES}

[1] L.V. Keldysh, Sov. Phys. Solid State 4, 1658 (1962).

[2] L. Esaki and R. Tsu, IBM J. Res. Dev. 14, 61 (1970); P. Lebwohl and R. Tsu, J. Appl. Phys. 42, 2664 (1970).

[3] M.I. Ovsyannikov, Yu.A. Romanov, V.N. Shabanov, and P.G.Loginova, Sov. Phys. Semicond. 4, 1919 (1970); Yu.A. Romanov, Sov. Phys. Semicond. 5, 1256 (1971).

[4] F. Bloch, Z. Phys. 52, 555 (1928); C. Zener, Proc. R. Soc. London Ser. A 145, 523 (1934).

[5] J. Feldmann, et al., Phys. Rev. B 46, 7252 (1992); K. Leo, et al., Solid State Commun. 84, 943 (1992); C. Waschke, et al., Phys. Rev. Lett. 70, 3319 (1993); V.G. Lyssenko, et al., Phys. Rev. Lett. 79, 301 (1997); M. Sudzius, et al., Phys. Rev. B 57, R12693 (1998); F. Lösser, et al., Phys. Rev. B 61, R13373 (2000).

[6] S.A. Ktitorov, G.S. Simin, and V.Ya. Sindalovsky. Sov. Phys. Solid State 13, 2230 (1971).

[7] H. Kroemer, cond-mat/0007482; cond-mat/0009311.

[8] A.A. Ignatov and Yu.A. Romanov, Phys. St. Solidi B73, 327 (1976).

[9] G.H. Wannier, Phys. Rev. 117, 432 (1950); Rev. Mod. Phys. 34, 645 (1962).

[10] Yu.A. Romanov and J.Yu. Romanova, JETP 91, 1033 (2000).

[11] A.A. Andronov and V.A. Kozlov, JETP Lett. 17, 87 (1973); Yu.V. Gulyaev and I.I. Chusov, Sov. Phys. Solid State 20, 1524 (1978); A. Matulis and A. Chenis, JETP 77, 1134 (1979).

[12] L.E. Vorob'ev, S.N. Danilov, V.N. Tulupenko, and D.A. Firsov, JETP Lett. 73, 219 (2001).

[13] Yu.A. Romanov, J.Yu. Romanova, L.G. Mourokh, and N.J.M.Horing, to be published.

[14] Y.C. Chang and R.B. James, Phys. Rev. B 39, 12672 (1989).

[15] L.K. Orlov and Yu.A. Romanov, Sov. Phys. Semicond. 19, 1157 (1985); Izv. Vusov, Radiofizika 32, 282 (1989) [in Russian]. 

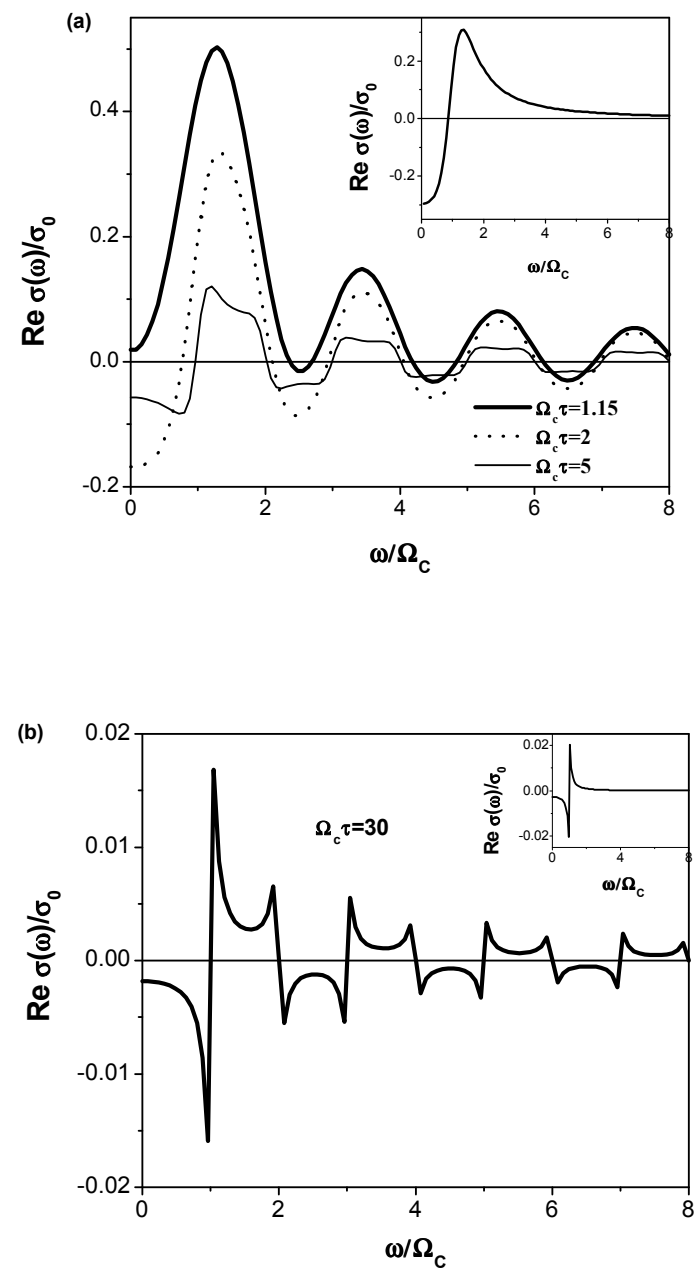

Yu.A.Romanov, et al., "Negative high-frequency...", Figure 1(a,b) of 5. 

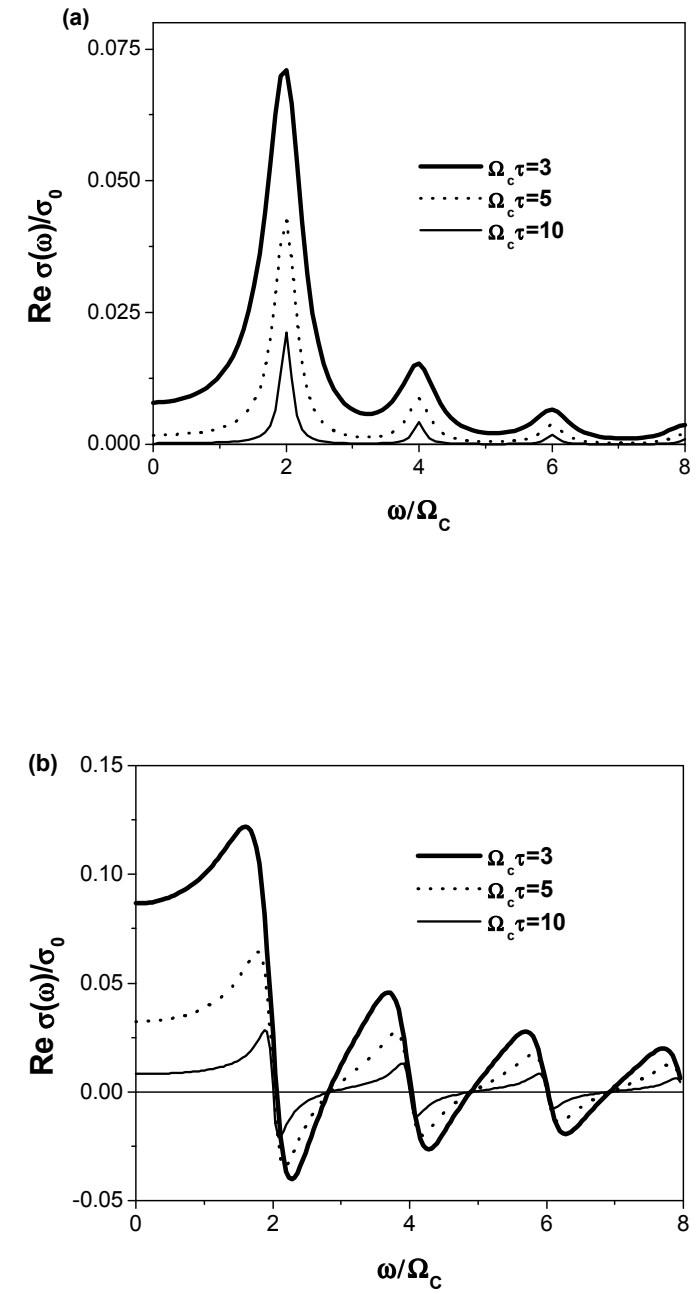

Yu.A.Romanov, et al., "Negative high-frequency...", Figure 2(a,b) of 5. 

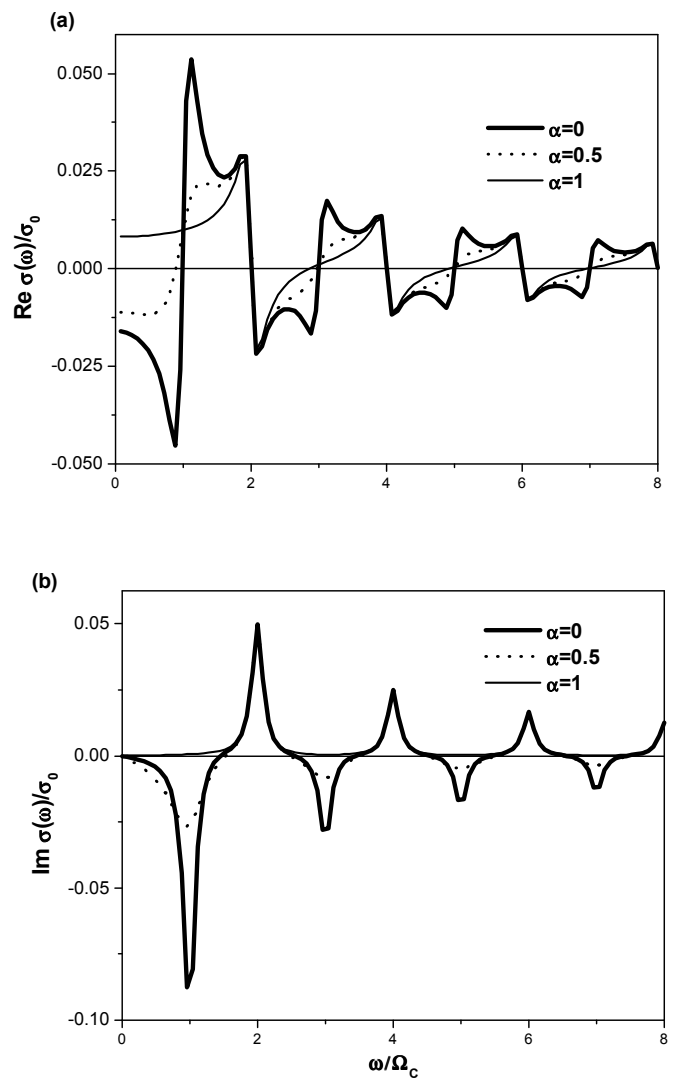

Yu.A.Romanov, et al., "Negative high-frequency...", Figure 3(a,b) of 5. 


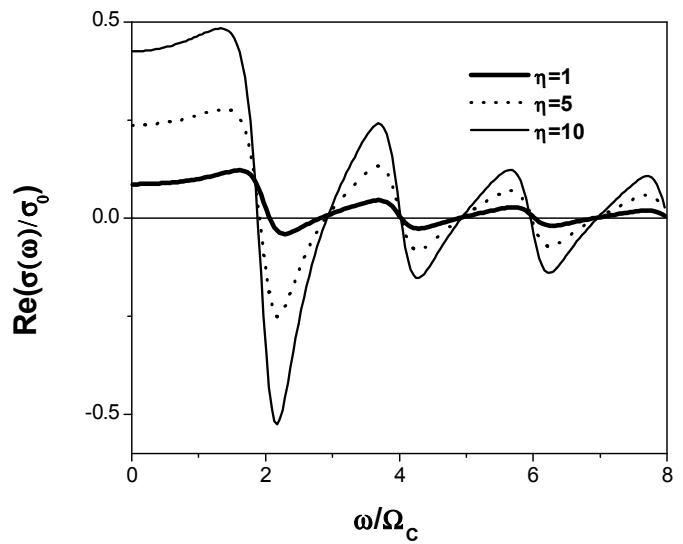

Yu.A.Romanov, et al., "Negative high-frequency...", Figure 4 of 5. 


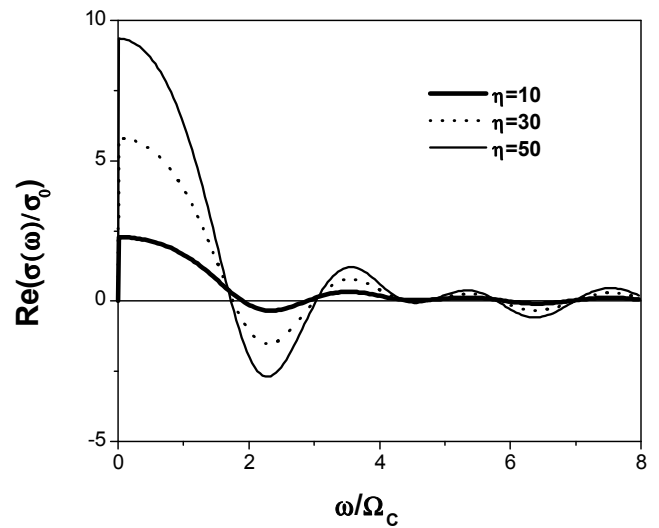

Yu.A.Romanov, et al., "Negative high-frequency...”, Figure 5 of 5. 